\title{
Synthesis and Properties of Polymethyl Methacrylate/Nanodiamond Composite Material
}

\author{
Lifang Ma ${ }^{1,2, a}$, Guoliang Cui ${ }^{1, a}$ Chunjing Tao ${ }^{2, b} H_{e p i n} \mathrm{Yan}^{2, b}$, Xiaogang $\mathrm{Hu}^{3, \mathrm{c}}$, \\ ${ }^{1}$ Beijing Institute of Technology , Beijing 100081 China \\ ${ }^{2}$ National Research Center for Rehabilitation Technical Aids , Beijing 100176 China \\ ${ }^{3}$ Beijing Grish Hitech Co., Ltd., Beijing 100085 China
}

aemail: malifang1105@163.com, bemail:664530199@qq.com, cemail: huxiaog922@163.com

\begin{abstract}
Keywords: polymethyl methacrylate; nanodiamond; synthesis
Abstract. Here, the nanodiamonds modified by different amounts of silane coupling agent were dispersed into the polymethyl methacrylate (PMMA) prepolymer. The PMMA/nanodiamond composite material was then synthesized by a room-temperature curing reaction. The dispersity of the nanodiamond in the composite materials was studied. The properties of the as-prepared composite materials were also analyzed. The results indicated that when the nanodiamond concentration was less than 1.0\%, the modified nanodiamond could be dispersed into the PMMA prepolymer homogeneously, which enhanced the mechanical properties of the PMMA composite materials. For the nanodiamond concentration of $1.0 \%$, the mechanical properties could be enhanced by over $1.0 \%$, which provided a new approach for improving the properties of PMMA-based composite materials.
\end{abstract}

\section{Introduction}

Polymethyl methacrylate (PMMA) is a widely used polymeric material in the fields of aerospace, machinery, medical and architecture industries. It has excellent properties such as small chromatic dispersion, high transmittance, great hardness, good chemical stability and good processing performance. Especially, the modified PMMA-based composite material has been used to prepare the base material for teeth and the prosthetic materials, which are commonly used in medical fields in recent years. The preparation of these composite materials is different from the conventional preparation methods. The room-temperature curing reaction method is applied to prepare the composite materials after the prepolymer is modified [1].

However, the composite materials prepared by a room-temperature curing reaction have problems such as relatively poor mechanical properties and unstable thermal properties. Introducing nanoparticles into the PMMA matrix to form polymer-based nano composite materials can combine the advantages of both organic and inorganic materials and improve the properties of PMMA. For example, NaWang et al., studied the influence of nanoparticles with different structures on the properties of PMMA-based composite material [2]. ShiboLi et al., used complex zirconia nanoparticles to reinforce the dental material [1]. The nanodiamond has excellent biocompatibility, mechanical properties and thermal conductivity. There have been studies on the preparation of the resin-based nanodiamond composite materials [3-5]. To the best of our knowledge, the introduction of nanodiamond into the PMMA-based composite materials has not been reported. In this work, the nanodiamonds modified by different amounts of silane coupling agent were dispersed into the PMMA prepolymer. The PMMA/nanodiamond composite material was then synthesized by a room-temperature curing reaction. The dispersity property of nanodiamond in the PMMA matrix was then studied and the properties of the obtained composite material were analyzed. 


\section{Experimental}

\subsection{Materials}

Methyl methacrylate (MMA, Tianjin Fuchen Chemical Reagent Factory) was purified by vacuum distillation before use. Benzoyl peroxide (BPO, Tianjin Fuchen Chemical Reagent Factory), N,N-dimethylaniline (Tianjin Fuchen Chemical Reagent Factory) and nanodiamond modified by silane coupling agent (Beijing Guoruisheng Technology Co., Ltd. ) were used as received.

\subsection{Methods}

\subsubsection{Preparation of the modified PMMA prepolymer}

A four-necked flask was set as the polymerization reactor. An electric stirrer, a thermometer, a reflux condenser and a nitrogen inlet were inserted into the four necks of the flask. The MMA and BPO were added into the flask under the dry nitrogen gas to complete the polymerization at $70{ }^{\circ} \mathrm{C}$ to obtain the preploymer. After the prepolymer was cooled to room temperature, some N, N-dimethylaniline was added to it and the mixture was stirred continuously. Finally, the modified PMMA prepolymer was obtained.

2.2.2. Dispersion of nanodiamond into the PMMA matrix

The modified PMMA prepolymer was mixed with different mass concentrations $(0.1 \%, 0.2 \%, 0.3 \%$, $0.5 \%, 0.75 \%$ and $1 \%$ ) of nanodiamond by a stirring-defoaming machine under ultrasonic stimulation. The product was filtered using a sieve to obtain the precursor for the curing reaction.

2.2.3. Preparation of the composite materials

The precursor for the curing reaction and the curing agent were added into the mold with a specific specification. The PMMA/nanodiamond composite material was obtained by the curing reaction at room temperature.

\subsection{Characterization}

\subsubsection{Electron Microscopy (SEM and TEM)}

The samples were placed into liquid nitrogen for $30 \mathrm{~min}$ and then quenched to break. The samples were then sprayed by gold and were analyzed by a Hitachi S-4800 scanning electron microscope with an acceleration voltage of $20 \mathrm{kV}$. The sliced samples were studied by a FEI $300 \mathrm{kV}$ field emission TEM.

2.3.2. Fourier transforms infrared spectroscopy (FTIR)

The analyzed pellet was made by the composite material and $\mathrm{KBr}$. The pellet was characterized by a Shimadzu Iffinity-21 Fourier transform infrared spectrometer at a resolution of $4 \mathrm{~cm}-1$ with a scan number of 16.

2.3.3. Characterization of the mechanical properties of the base material

The WDT-type universal testing machine (made in China) was used to measure the flexural strength and elastic modulus of the samples. According to GB/T 1449-2005 standard, the specimen span of 34 $\mathrm{mm}$ and the loading speed of $5 \mathrm{~mm} / \mathrm{min}$ was used in the three-point bending test. The micro hardness of the composite material was measured by the HXD-TM micro hardness tester (made in China).

\section{Results and Discussion}

\subsection{Observation on stability of the nanodiamond}

The materials for the curing reaction with various nanodiamond concentrations were placed for seven days to observe their standing stability. For the material with nanodiamond concentration over 1\%, an obvious separation appeared. The separation phenomenon was still present despite the blending of the material, as shown in Figure 1 . This showed that the material for the curing reaction with nanodiamond concentration over1.0\% did not have a long-term stability, as the higher nanodiamond concentration hindered the uniform dispersion of the nanodiamond. Therefore, the material for the curing reaction with nanodiamond concentration over $1.0 \%$ was not suitable for further preparations 
of the composite materials.

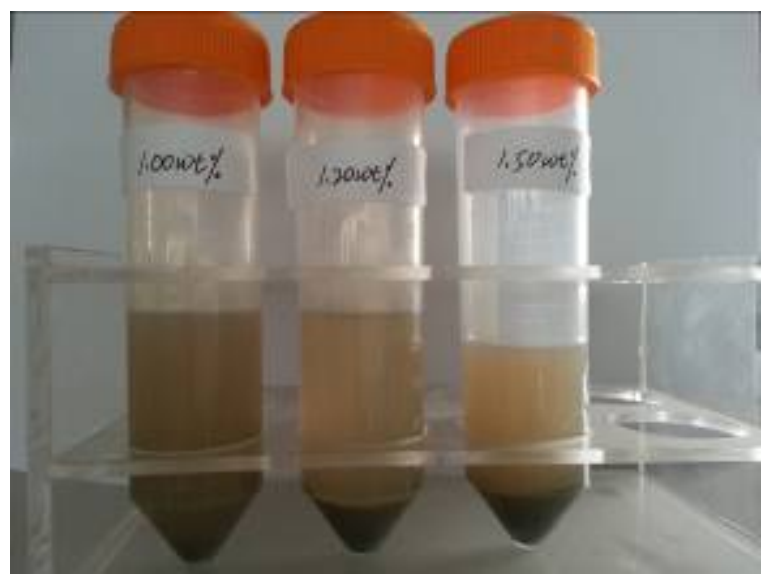

Fig 1. The picture of the composite materials for the curing reaction with different amounts of nanodiamond after the composite materials were placed for seven days.

\subsection{Electron Microscopy}
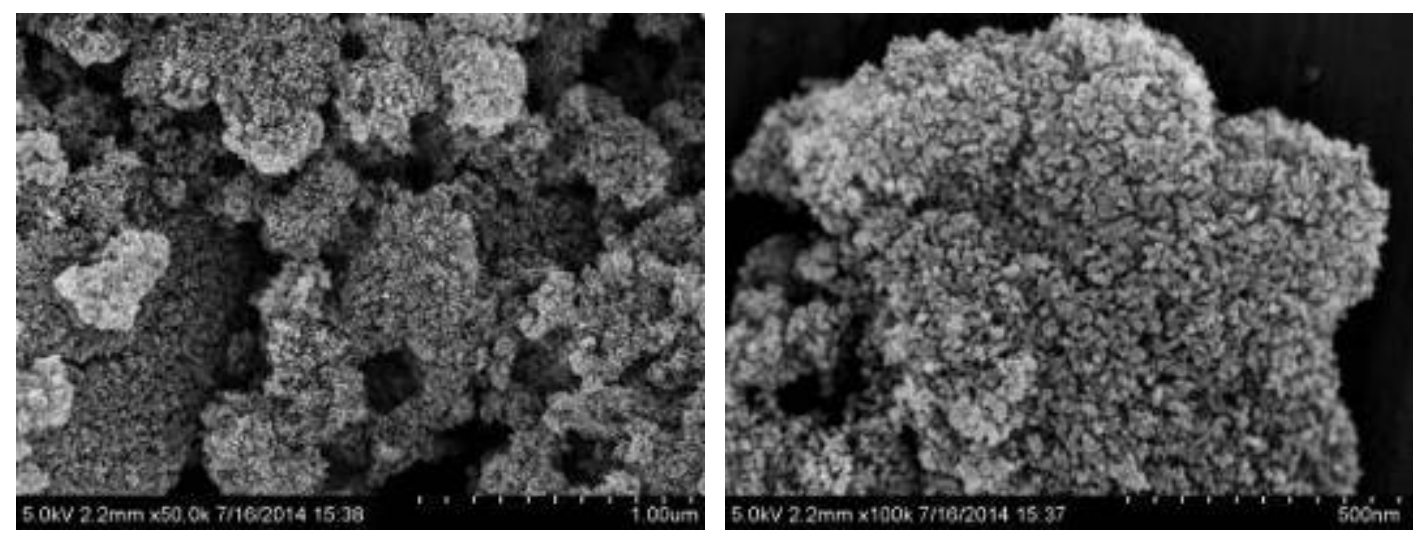

Fig 2 .SEM images of the modified nanodiamond.
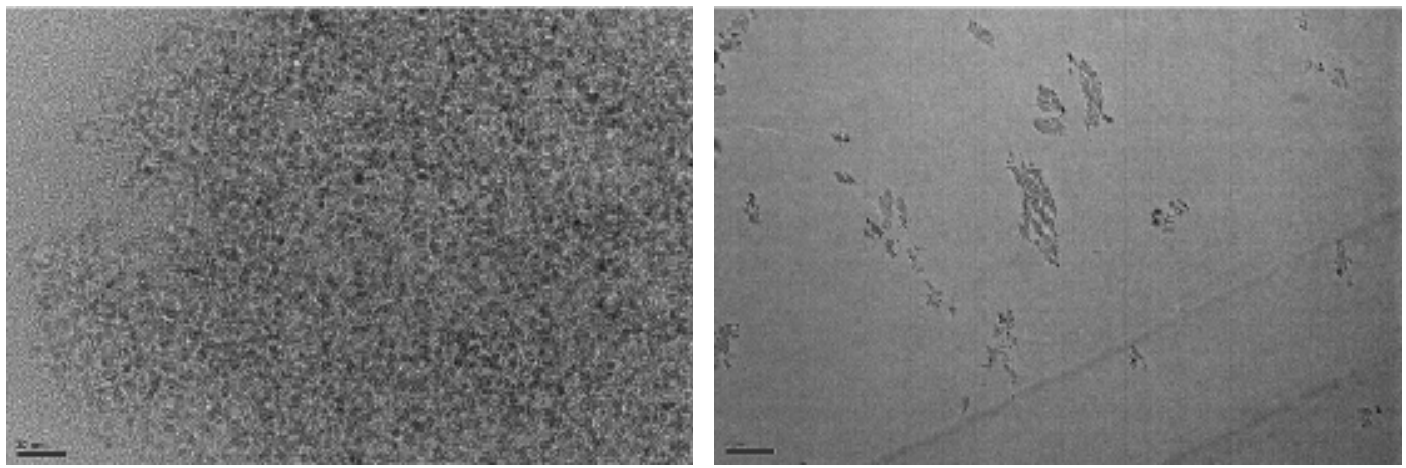

Fig 3. TEM images of PMMA/nanodiamond composite materials.

Figure 2 shows the SEM images of the nanodiamond modified by the silane coupling agent. The nanodiamond modified by the silane coupling agent shows a spherical shape with a single crystal size of around $10 \mathrm{~nm}$ and a relatively large specific surface area. Figure 3 shows the TEM images of the dispersity of nanodiamond in the matrix material. The nanodiamond particles are shown to be loosely dispersed in the PMMA and the distance between the particles and that between the agglomerates are both relatively large. Meanwhile, according to the microstructure, the resin matrix has infiltrated into the gap between the ultrafine agglomerates of diamond and almost fully fills the gaps between the 
diamond particles. Therefore, the interaction between diamond agglomerates and resin matrix not only has the microscopic combination through chemical bonding but also involves the macroscopic mechanical interlocking, which can reinforce the integration between these two components.

\subsection{FTIR Spectra of PMMA/Nanodiamond Composite Material}

FTIR tests were implemented on the PMMA composite materials with and without nanodiamond, see Figure 4 and Figure 5. The relatively broad absorption peaks at $2950 \mathrm{~cm}-1$ correspond to symmetric and antisymmetric stretching vibration of $\mathrm{C}-\mathrm{H}$ bonds in - $\mathrm{CH} 3$ and -CH2- groups. With the addition of nanodiamond, the peak intensity becomes higher and the peak becomes wider, due to the effect of silane coupling agent on the nanodiamond. The peaks at $1147 \mathrm{~cm}-1,1190 \mathrm{~cm}-1,1240 \mathrm{~cm}-1$ and 1270 $\mathrm{cm}-1$ are attributed to stretching vibration of $\mathrm{C}-\mathrm{C}-\mathrm{O}-\mathrm{C}$, which have no significant difference from the characteristic bands of poly (methyl methacrylate) material. However, the peak at $1435 \mathrm{~cm}-1$ presents an obvious enhancement, which verifies the combined interaction of the deformation vibration of Si-CH2 bonding. The intensity of the peak at $1720 \mathrm{~cm}-1$ corresponding to stretching vibration of $\mathrm{C}=\mathrm{O}$ also increases with the addition of nanodiamond. In the spectra, the absorption peaks in the range of 700-900 cm-1 are attributed to the stretching vibration absorption of Si-C bond. The peak around $950 \mathrm{~cm}-1$ is attributed to the stretching vibration of Si-O-C bond and the one around 1050 $\mathrm{cm}-1$ is attributed to the stretching vibration absorption of Si-O-Si bond. These peaks confirm the presence of silane coupling agent and also indicate that the silane has been grafted to the surface of nanodiamond particles through the Si-O-C bond. The FTIR spectra show that the silane coupling agent molecules are grafted to the surface of nanodiamond by hydrogen bonding. Moreover, the polymerization between the coupling agent molecules produces Si-O-Si bonds, which form a layer of mesh structure on the surface of nanodiamond. In addition, the hydroxyl groups of matrix material may interact with the carbonyl group of the silane coupling agent by hydrogen bonding, which can connect the matrix material with the nanodiamond indirectly[6-8].



Figure 4. FTIR spectra of PMMA material. 




Figure 5. FTIR spectra of PMMA/nanodiamond composite material.

\subsection{Mechanical Properties of PMMA/Nanodiamond Composite Materials}

Table 1 shows the comparison of mechanical properties of the base materials with and without nanodiamond. It is shown that after the base material with nanodiamond was cured, its mechanical properties were enhanced. When the nanodiamond concentration was $1 \%$, the enhancement had exceeded $10 \%$. The main reason for the enhancement was that the nanodiamond modified by silane coupling agent had a bigger steric hindrance and a stronger interaction with the macromolecules of the base material, which could make the nanodiamond in matrix material more stable. After the addition of the curing agent, the $\mathrm{C}=\mathrm{C}$ double bond and the hydrogen bond in both the silane coupling agent molecules on the surface nanodiamond and the base material molecules can be activated due to the effect of the initiator. Then, the activated bonds can randomly form some new chemical bonds with adjacent double bond and hydrogen bond, which are interwoven and self-polymerized to generate three-dimensional network structures. The nanodiamond particles are combined with the base material by chemical bonds and network structure, which can enhance the mechanical properties of the cured base materials[7].

Table 1. Mechanical properties of the composite materials with different contents of nanodiamond.

\begin{tabular}{|c|c|c|c|c|}
\hline $\begin{array}{c}\text { Nanodiamond } \\
\text { content }\end{array}$ & $\begin{array}{c}\text { Compression } \\
\text { strength }\end{array}$ & $\begin{array}{c}\text { Tensile } \\
\text { Strength/MPa }\end{array}$ & $\begin{array}{c}\text { Elastic } \\
\text { modulus/MPa }\end{array}$ & micro hardness \\
\hline 0 & $72.38 \pm 1.37$ & $40.26 \pm 1.86$ & $996.11 \pm 45.35$ & 9.16 \\
\hline $0.5 \%$ & $78.55 \pm 1.78$ & $44.07 \pm 1.49$ & $1127.87 \pm 78.71$ & 13.97 \\
\hline $1.0 \%$ & $82.42 \pm 1.55$ & $46.72 \pm 1.16$ & $1237.62 \pm 52.30$ & 16.29 \\
\hline
\end{tabular}

\section{Conclusions}

This study indicated that the nanodiamond can be dispersed properly into the PMMA matrix by using a room-temperature curing reaction. When the nanodiamond concentration was less than $1.0 \%$, the modified nanodiamond can be dispersed in the PMMA prepolymer homogenously and can enhance the mechanical properties of the PMMA composite material. According to our results, the mechanical properties can be enhanced by over $10 \%$, when the nanodiamond concentration is $1 \%$. Our study 
provides a new approach for improving the properties of the PMMA-based composite materials.

\section{References}

[1] Na Wang,Na Gao, ,Hongwei Li , Qinghong Fang , Erfan Chen. Influence of Different Structural Particle on the Properties of PMMA Composites [J]. ACTA CHIMICA SINICA, 2010, 68(8): 827-832.

[2] ShiboLi, Zhongyi Wang,Zhaohui Chen,Haifeng Hu,LihuiTang,Chufan Ma.The study on fabrication of dental restoration using PMMA- ZrO2 composites via CAD/ CAM[J]. Chin J Stomatol,, 2005, 40(1): 23-25.

[3] Wei Yu, Huaqing Xie, Yang Li, Lifei Chen, Qiang Wang. Experimental investigation on the thermal transport properties of ethylene glycol based nanofluids containing low volume concentration diamond nanoparticles [J]. Colloids and Surfaces A: Physicochem. Eng. Aspect, 380(2011)1-5.

[4] Huaqing Xie, Wei Yu, Yang Li. Thermal performance enhancement in nanofluids containing diamond nanoparticles [J]. Journal of Physics D: Applied Physics, 42(2009)095413.

[5] Yaqing Chu, Yi Tong, Fenglei Huang, Tonglai Zhang. Structure and properties of boundary layer between nanodiamond and resin matrix [J]. Journal of Beijing Institute of Technology, 2013, 33(1): $1-5$.

[6] Yaqing Chu, Yi Tong, Fenglei Huang, Tonglai Zhang. Mechanical properties of dental composite resins with nanodiamonds of different diameters [J]. Journal of Beijing Institute of Technology, 2012, 21(1): 19-22.

[7] Chu Y Q, Tong Y, Huang F l, Zhang T L. Synthesis of nanodiamond reinforced dental composite resins and their mechanical properties [J]. Fullerenes, Nanotubes, and Carbon Nanostructures, 2012, 20(1/8): 628-632.

[8] Xiaogang Hu, Xiaoyu Gu, Yi Tong, Zengming Zhang, Yunjun Luo, Fenglei Huang, Xinzhi Wang. Study on modified ultrafine-diamond reinforcing and toughening bis-phenol A dental composites [J]. New Chemical Materials, 2006, 34(2): 60-62. 